\title{
Quantum decay of metastable states in small magnetic particles
}

\author{
M.-Carmen Miguel and Eugene M. Chudnovsky* \\ Departament de Física Fonamental, Facultat de Física, Universitat de Barcelona, Diagonal 647, E-08028 Barcelona, Spain
}

(Received 21 August 1995; revised manuscript received 16 February 1996)

\begin{abstract}
We present an imaginary-time path-integral study of the problem of quantum decay of a metastable state of a uniaxial magnetic particle placed in the magnetic field at an arbitrary angle. Our findings agree with earlier results of Zaslavskii obtained by mapping the spin Hamiltonian onto a particle Hamiltonian. In the limit of low barrier, weak dependence of the decay rate on the angle is found, except for the field which is almost normal to the anisotropy axis, where the rate is sharply peaked, and for the field approaching the parallel orientation, where the rate rapidly goes to zero. This distinct angular dependence, together with the dependence of the rate on the field strength, provides an independent test for macroscopic spin tunneling. [S0163-1829(96)05625-1]
\end{abstract}

\section{INTRODUCTION}

Small magnetic particles may have two or more equilibrium orientations of the magnetic moment. In the presence of a magnetic field only one of them corresponds to the absolute minimum of the energy; the others are metastable. They decay with time due to thermal overbarrier transitions. As the temperature goes down, thermal transitions die out but a finite probability of quantum transitions remains. ${ }^{1-3}$ In such transitions the uncertainty principle manifests itself on a scale where it has been rarely seen. As many as $10^{6}$ spins can coherently tunnel out of a metastable magnetic state, placing this effect in the domain of macroscopic quantum tunneling (MQT). ${ }^{4}$

Any disturbance of the ferromagnetic order on a scale less than the domain wall thickness (typically $\sim 100-1000 \AA$ ) costs large exchange energy. For a nanometer scale particle, a stiff magnetic moment $\vec{M}$ that can rotate but not change its absolute value is believed to be a good approximation. When such a particle is embedded in a nonmagnetic matrix, $\vec{M}$ interacts weakly with the microscopic degrees of freedom: phonons, conducting electrons, nuclear spins, etc. The influence of these interactions on the tunneling rate can be noticeable ${ }^{5-9}$ but, apparently, does not kill the effect. Experiments have been reported ${ }^{10-17}$ which indicated the possible presence of magnetic tunneling in small particles and in large magnetic molecules. Applications of this phenomenon have been discussed which include the reliability of small magnetic units in memory devices and their use in quantum computers. ${ }^{18}$ Beautiful topological interference effects have been suggested, which have no analogy in other MQT problems. ${ }^{1920}$ All this makes magnetic tunneling an exciting area for theoretical research and a challenging experimental problem.

Experiments performed to date were done on ensembles of small particles or on individual particles of large size. Both systems exhibit stochastic behavior that precludes them from direct comparison with theory. It is likely, however, ${ }^{13}$ that in the nearest future measurements of individual nanometer particles will become possible. The simplest choice would be a uniaxial magnetic particle whose magnetic anisotropy can be approximated by $E_{\text {an }}=-k M_{z}^{2}$. This model, however, does not possess tunneling until a term is added to the energy (e.g., $k^{\prime} M_{x}^{2}$ ) that violates its commutation with $M_{z}$. A number of models have been studied, ${ }^{1-3}$ which consider a more complex (than uniaxial) structure of the magnetic ansitropy. The generic problem, however, and the easiest to implement in practice, is the one of uniaxial anisotropy and the magnetic field applied at a some angle $\theta_{H}$ with the anisotropy axis. This problem does not possess any symmetry and for that reason is more difficult mathematically. It was first studied by Zaslavskii ${ }^{21}$ within the approach ${ }^{22}$ that maps the spin problem onto a particle problem. The corresponding particle Hamiltonian, while different from the spin Hamiltonian, has the same structure of low-lying energy levels. Using this method and the semiclassical approximation, Zaslavskii calculated the tunneling exponent, the preexponental factor, and their temperature dependences in the limit of low barrier. In this paper we shall study the uniaxial case by direct calculation of the imaginary-time path integral for the magnetic moment. ${ }^{1-3}$ For the problem at hand, both methods make a number of approximations; the exact solution is impossible to obtain. One may worry, therefore, how reliable their predictions are for the dependence of the tunneling rate on the magnitude and the orientation of the field. In Sec. III, we shall demonstrate that angular and field dependences of the tunneling exponent obtained by Zaslavskii's method and by the path-integral method coincide precisely. The tunneling exponent must be robust with respect to the exact positions of spin energy levels. On the contrary, the prefactor for tunneling between nonequivalent wells must be strongly affected by the positions and widths of the levels (see Sec. IV). Fortunately, the exponent is the easiest to measure. Our purpose is to analyze the problem in terms of observable variables, to make a connection with previously studied tunneling problems, to establish the range of validity of the semiclassical approximation, and to make suggestions for future experiments.

The solution of this problem elucidates some important points with regard to the two models which have been discussed in literature: with the field parallel $^{3}$ and perpendicular ${ }^{1-3}$ to the anisotropy axis. We will show that even a very small misalignment of the field with the above two orientations completely changes the results. For the strictly uniaxial case no tunneling is expected when the field 


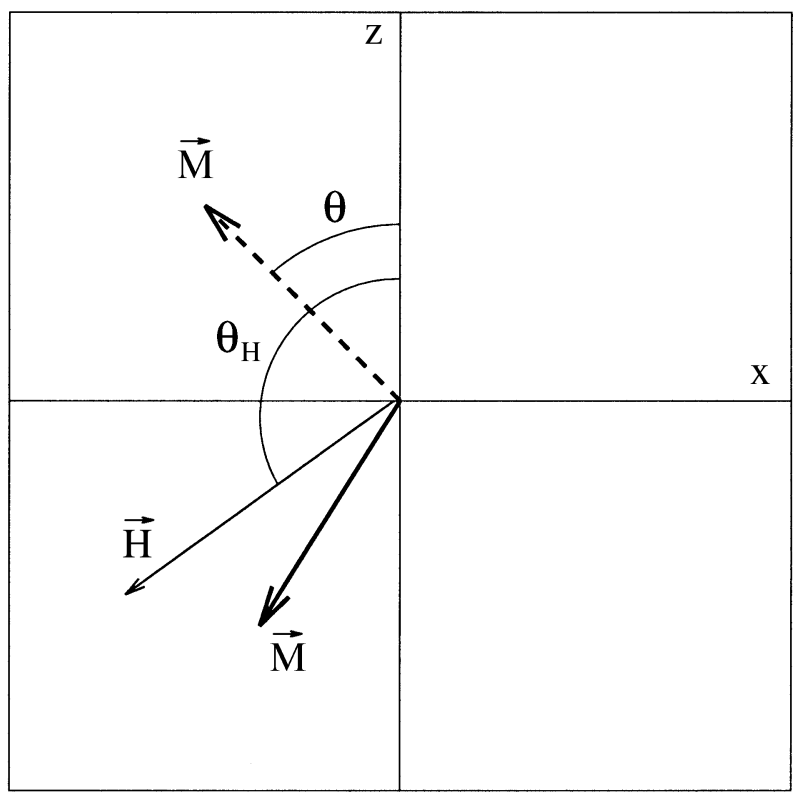

FIG. 1. The geometry of the problem. Vectors $\vec{M}$ shown by dashed and solid lines correspond to the metastable and stable states, respectively.

is parallel to the anisotropy axis. It turns out, however, that the tunneling exponent $B$ depends on $\theta_{H}$ via $\left|\cot \theta_{H}\right|^{1 / 6}$. Correspondingly, to notice the freezing of a metastable state at $\theta_{H}=180^{\circ}$, the field must be well aligned with the anisotropy axis. Another interesting conclusion concerns the field dependence of $B$. For particles of a considerable size, $H$ must be close to the critical field $H_{c}$ at which the barrier disappears; otherwise the lifetime with respect to quantum decay of a metastable state becomes exponentially large. The small parameter of the theory is $\epsilon=1-H / H_{c}$. In a wide range of angles $B \propto \epsilon^{5 / 4}$, not $\epsilon^{3 / 2}$ as was previously obtained for some special symmetric cases. The crossover from asymmetric to symmetric tunneling will be discussed in Sec. III. For measurements of a single particle, $B \sim 30-35$ should be optimal (see Sec. V). At $\epsilon \sim 10^{-3}$ this would correspond to tunneling of a total spin $S \sim 30000$ by an angle of more than $4^{\circ}$ on a time scale of a few hours. We shall argue in Sec. III that the semiclassical approximation must be rather good in this case. The prefactor of the tunneling rate and the crossover from quantum to thermal regime will be discussed in Sec. IV. Besides small particles, some of our predictions can be tested in $M n_{m}$ molecular complexes which have strong uniaxial magnetic anisotropy (Sec. V). In this case, a greater $B$ and, correspondingly, a much longer lifetime are anticipated. ${ }^{14}$

\section{FORMULATION OF THE PROBLEM}

Consider a uniaxial single-domain particle whose anisotropy direction coincides with the $z$ axis. In the absence of the field there are two equilibrium orientations of $\vec{M}$ : along and against the $z$ direction. Let us now assume that the magnetic field is applied in the $Z X$ plane, at an angle $90^{\circ} \leqslant \theta_{H} \leqslant 180^{\circ}$ with the $z$ axis. If the field is below some critical value $H_{c}\left(\theta_{H}\right)$ (to be computed), $\vec{M}$ has two equilibrium orientations shown in Fig. 1. One of them, with

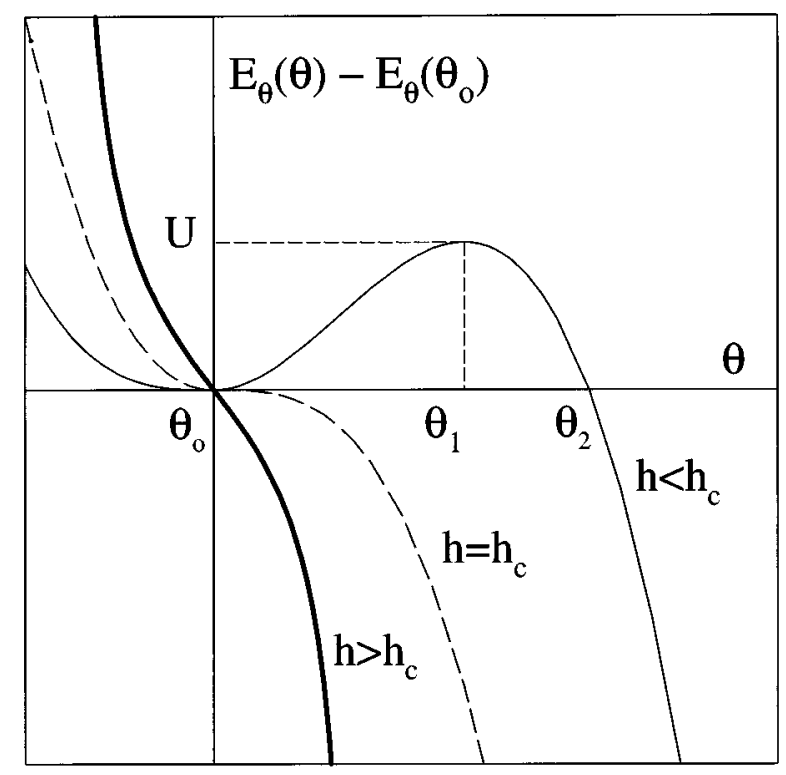

FIG. 2. The $\theta$ dependence of the potential for different values of the field.

$0^{\circ} \leqslant \theta_{H} \leqslant 90^{\circ}$, is a metastable state, while another, with $90^{\circ} \leqslant \theta_{H} \leqslant 180^{\circ}$, corresponds to the absolute minimum of the magnetic energy. We are interested in the quantum decay of the metastable state.

The magnetic energy of the particle is a sum of its anisotropy energy and Zeeman energy,

$$
E=-k M_{z}^{2}-M_{x} H_{x}-M_{z} H_{z},
$$

where $k>0$ is a dimensionless anisotropy constant. For an arbitrary orientation of $\vec{M}$ the energy can be rewritten as

$$
E=-H_{a} M\left(\frac{1}{2} \cos ^{2} \theta+h_{z} \cos \theta+h_{x} \sin \theta \cos \phi\right),
$$

where we have introduced the anisotropy field $H_{a}=2 k M$ and dimensionless components of the magnetic field, $h_{x, z}=H_{x, z} / H_{a}, \theta$ and $\phi$ being the conventional spherical coordinates of the fixed-length vector $\vec{M}$. The metastable state exists at $H_{z}<0$. It corresponds to $\vec{M}$ in the $X Z$ plane (that is, $\phi=0$ ) at a some angle $\theta=\theta_{0}$. Near this point the potential has the form of a canyon with the bottom at $\phi=0$ satisfying $E=\left(H_{a} M\right) E_{\theta}$, where

$$
E_{\theta}=-\frac{1}{2} \cos ^{2} \theta-h \cos \left(\theta-\theta_{H}\right) .
$$

Here we have switched to $\theta_{H}$ and $h=H / H_{a}$ from $h_{x}=h \sin \theta_{H}$ and $h_{z}=h \cos \theta_{H}$. The dependence of $E_{\theta}$ on $\theta$, near $\theta_{0}$, at different values of the field, is schematically shown in Fig. 2.

The metastable state satisfies

$$
\sin 2 \theta_{0}+2 h \sin \left(\theta_{0}-\theta_{H}\right)=0,
$$

which follows from $\partial E_{\theta} / \partial \theta=0$. The point $\theta=\theta_{1}$ is the saddle point of the potential $E(\theta, \phi)$. For the metastable state to decay, $\vec{M}$ must either fluctuate to the top of the barrier at $\theta=\theta_{1}$ or tunnel under the barrier to the escape point $\theta=\theta_{2}$. Since $\vec{M}$ is considered to be a macroscopic 


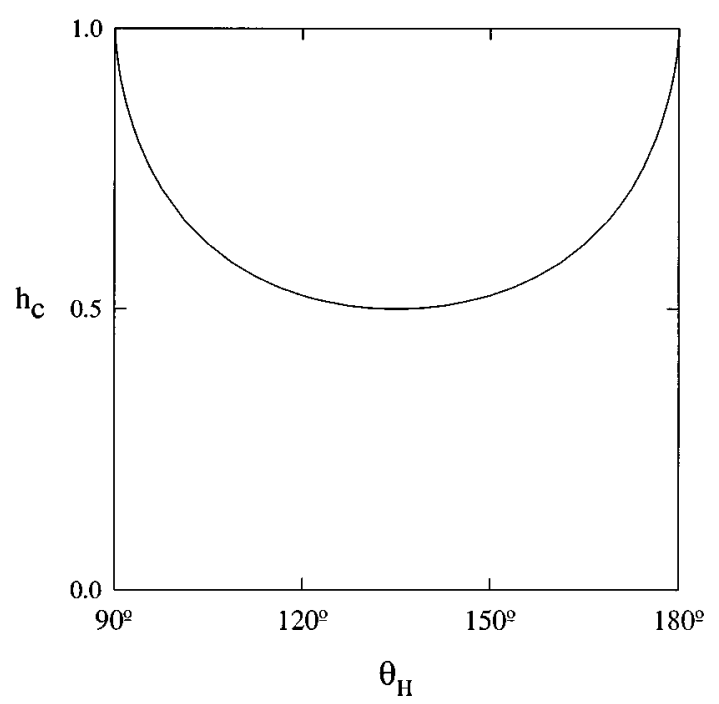

FIG. 3. The dependence of the critical value of the field on the field orientation.

variable, an appreciable escape rate is expected only when the barrier is lowered by tunneling the external field to the critical field $h_{c}\left(\theta_{H}\right)$. At $h=h_{c}$ all three angles in Fig. 2 coincide, $\theta_{0}=\theta_{1}=\theta_{2}=\theta_{c}$, and both the first and the second derivatives of $E_{\theta}$ become zero at $\theta=\theta_{c} .{ }^{23}$ This gives two equations for $\theta_{c}$ and $h_{c}$ :

$$
\begin{aligned}
& \sin 2 \theta_{c}+2 h_{c} \sin \left(\theta_{c}-\theta_{H}\right)=0, \\
& \cos 2 \theta_{c}+2 h_{c} \cos \left(\theta_{c}-\theta_{H}\right)=0 .
\end{aligned}
$$

Solving them we obtain

$$
\begin{gathered}
\sin ^{3} \theta_{c}=h_{c} \sin \theta_{H}, \\
\cos ^{3} \theta_{c}=-h_{c} \cos \theta_{H}, \\
h_{c}=\left(\sin ^{2 / 3} \theta_{H}+\left|\cos \theta_{H}\right|^{2 / 3}\right)^{-3 / 2} .
\end{gathered}
$$

Note that all signs in Eq. (6) are in accordance with the fact that $0^{\circ} \leqslant \theta_{c} \leqslant 90^{\circ}$ while $90^{\circ} \leqslant \theta_{H} \leqslant 180^{\circ}$. The dependence of $h_{c}$ on $\theta_{H}$ is plotted in Fig. 3.

Let us now consider the field that is slightly lower than the critical field,

$$
h=h_{c}(1-\epsilon),
$$

where $\epsilon \ll 1$. Such a field still preserves the metastable state. The corresponding equilibrium value of $\theta$ is now slightly lower than $\theta_{c}, \theta_{c}-\theta_{0}=\Delta \ll 1$. Expanding Eq. (4) near $\theta_{c}$ we obtain, with the help of Eqs. (5), $\Delta=(2 \epsilon / 3)^{1 / 2}$. Then introducing a positive $\delta=\left(\theta-\theta_{0}\right) \ll 1$, and expanding Eq. (3) near $\theta_{0}$, we get up to third order in $\delta$,

$$
E_{\theta}(\theta)-E_{\theta}\left(\theta_{0}\right)=\frac{1}{4} \sin 2 \theta_{c}\left(\sqrt{6 \epsilon} \delta^{2}-\delta^{3}\right) .
$$

The escape point is $\delta_{2}=\theta_{2}-\theta_{0}=\sqrt{6 \epsilon}$. This concludes our study of the classical problem and brings us to the problem of the quantum decay of the metastable state described by Eq. (9).

\section{COMPUTATION OF THE TUNNELING EXPONENT}

The tunneling rate can be written as $\Gamma=A \exp (-B)$, with the exponent $B$ being determined by the imaginary time action $^{1-3,19,20}$

$$
I=\int d \tau\left(i \frac{M}{\gamma} \dot{\phi}_{\tau}(1-\cos \theta)+E(\theta, \phi)\right),
$$

where $\tau=i t, \gamma$ is the gyromagnetic ratio, and $E(\theta, \phi)$ is given by Eq. (2). Introducing $\omega_{a}=\gamma H_{a}$, the dimensionless imaginary time $\bar{\tau}=\omega_{a} \tau$, and the total spin of the particle, $S=M / \hbar \gamma \gg 1$, we get

$$
\begin{aligned}
I= & -\hbar S \int d \bar{\tau}\left[i \dot{\phi}_{\bar{\tau}}(\cos \theta-1)+\frac{1}{2} \cos ^{2} \theta+h_{z} \cos \theta\right. \\
& \left.+h_{x} \sin \theta \cos \phi\right] .
\end{aligned}
$$

To obtain the tunneling exponent, one must consider the extremal trajectories of Eq. (11):

$$
\begin{gathered}
i \dot{\theta}_{\bar{\tau}}=h_{x} \sin \phi, \\
i \dot{\phi}_{\bar{\tau}} \sin \theta=h_{x} \cos \theta \cos \phi-h_{z} \sin \theta-\cos \theta \sin \theta
\end{gathered}
$$

Here $\phi$ plays the role of the coordinate while $\cos \theta$ plays the role of the momentum. The latter is imaginary under the barrier, which explains the presence of $i$ in Eq. (12). Note that, as usual, the generalized coordinate and momentum can be interchanged in our calculation. Instead of considering real $\phi$ and imaginary $\cos \theta$, one can consider imaginary $\phi$ and real $\theta$. In fact, the latter is the only appropriate choice because, according to the chosen geometry (see Fig. 1), $\theta$ is the real tunneling coordinate while $\phi=0$ in both initial and final states.

Equations (12) have a solution (an instanton) that carries out the underbarrier rotation of $\vec{M}$ to the escape point. For this solution both $\theta$ and $\phi$ depend on $\tau$. It starts at $\theta=\theta_{0}$, $\phi=0$ at $\tau=-\infty$, comes to $\theta=\theta_{2}, \phi=0$ at $\tau=0$, and then bounces back to $\theta=\theta_{0}, \phi=0$ at $\tau=+\infty$. The general solution is difficult to obtain. We shall recall that we are interested in the case of low barrier when $h \rightarrow h_{c}$. In this case the potential in Fig. 2 becomes nearly flat and the $\bar{\tau}$ derivative of $\theta$ must be proportional to a some power of a small parameter $\epsilon$. This is the approximation of a slow instanton, which is easy to understand if one notices that the instanton corresponds to the classical motion in the inversed potential (which is now almost flat). According to the first of Eqs. (12), this means that the instanton involves only small $\phi$; that is, the classical trajectory lies near the bottom of the potential canyon, close to the $X Z$ plane. The phase term in Eq. (11), proportional to $\dot{\phi}_{\bar{\tau}}$ (not the $\dot{\phi}_{\bar{\tau}} \cos \theta$ term), is important for tunneling between equivalent minima. ${ }^{19,20}$ For the closed instanton trajectory described above it gives a zero contribution to the integral and, therefore, can be omitted.

The tunneling exponent $B$ follows from the path integral

$$
\int \mathcal{D}\{\phi(\tau)\} \int \mathcal{D}\{\cos \theta(\tau)\} \exp \left(-\frac{I}{\hbar}\right)
$$

over the continuum of trajectories which start and end at the metastable state $\theta=\theta_{0}, \phi=0$ and which are close to the 
instanton. After integrating $\dot{\phi}_{\bar{\tau}} \cos \theta$ in Eq. (11) by parts, with account of the boundary condition $\phi( \pm \infty)=0$, and using the smallness of $\phi$ along the instanton, the path integral that we have to compute becomes

$$
\begin{aligned}
\int \mathcal{D}\{\phi(\tau)\} \int \mathcal{D}\{\cos \theta(\tau)\} \exp \left\{-S \int_{-\infty}^{\infty} d \bar{\tau}\left(-i \dot{\theta}_{\bar{\tau}}(\phi \sin \theta)\right.\right. \\
\left.\left.+\frac{h \sin \theta_{H}}{2 \sin \theta}(\phi \sin \theta)^{2}+E_{\theta}(\theta)\right)\right\},
\end{aligned}
$$

where $E_{\theta}(\theta)$ is given by Eq. (3). One can now select the new variables of the functional integration, $\phi \sin \theta$ and $\cos \theta$, and notice that the integration over $\phi \sin \theta$ is Gaussian. This gives

$$
B=S \int_{-\infty}^{\infty} d \bar{\tau}\left(\frac{\dot{\theta}^{2} \sin \theta_{c}}{2 h_{c} \sin \theta_{H}}+E_{\theta}(\theta)-E_{\theta}\left(\theta_{0}\right)\right) .
$$

Note that we put $\theta=\theta_{c}$ and $h=h_{c}$ in the first term because $\dot{\theta}_{\bar{\tau}}^{2}$ already has a smallness of order $\sqrt{\epsilon}$ coming from the $\bar{\tau}$ derivative, so that this term is proportional to $\epsilon^{3 / 2}$ and is of the same order in $\epsilon$ as other terms in Eq. (15) (justified below). A constant $E_{\theta}\left(\theta_{0}\right)$ is added to make $B=0$ at $\theta(\bar{\tau})=\theta_{0}$. The $\bar{\tau}$ integration must now be performed over the instanton $\theta(\bar{\tau})$ that minimizes $B$. This simplifies the problem tremendously, compared to the problem where the action depended on $\phi(\bar{\tau})$ and $\theta(\bar{\tau})$, though a complete mathematical equivalence to the initial problem is preserved. We shall now use the fact that in the limit of small barrier the instanton involves small deviations from $\theta_{0}$ and replace $\theta$ in Eq. (15) by $\theta_{0}+\delta$. With the help of Eq. (9) we obtain

$$
B=S \int_{-\infty}^{\infty} d \bar{\tau}\left(\frac{\dot{\delta}_{\bar{\tau}}^{2} \sin \theta_{c}}{2 h_{c} \sin \theta_{H}}+\frac{1}{4} \sin 2 \theta_{c}\left(\sqrt{6 \epsilon} \delta^{2}-\delta^{3}\right)\right) .
$$

The extremal trajectory of Eq. (16) is

$$
\delta(\tau)=\frac{\delta_{2}}{\cosh ^{2}\left(\omega_{0} \tau\right)}
$$

where

$$
\omega_{0}=\left(\frac{3}{8}\right)^{1 / 4} \epsilon^{1 / 4} \frac{\sin ^{1 / 2} \theta_{H}\left|\cos \theta_{H}\right|^{1 / 6}}{\sin ^{2 / 3} \theta_{H}+\left|\cos \theta_{H}\right|^{2 / 3}} \omega_{a} .
$$

Expressions (6) and (7) have been used to obtain the dependence of $\omega_{0}$ on $\theta_{H}$. As required, the instanton starts at $\delta=0\left(\theta=\theta_{0}\right)$ at $\tau=-\infty$, comes to the escape point $\delta=\delta_{2}$ ( $\left.\theta=\theta_{2}\right)$ at $\tau=0$, and then bounces back to $\delta=0\left(\theta=\theta_{0}\right)$ at $\tau=+\infty$. The substitution of Eq. (17) into Eq. (16) gives, with the help of Eqs. (6) and (7), a simple formula for $B$,

$$
B=\frac{16 \times 6^{1 / 4}}{5} S \epsilon^{5 / 4}\left|\cot \theta_{H}\right|^{1 / 6}
$$

Note that this expression can be obtained from (16) without knowing the explicit form of the instanton, Eqs. (17) and (18). It is instructing, however, to see from Eq. (17) how tunneling occurs via rotation of the magnetic moment in imaginary time. We shall also need the instanton frequency (18) for computing the crossover temperature in Sec. IV.

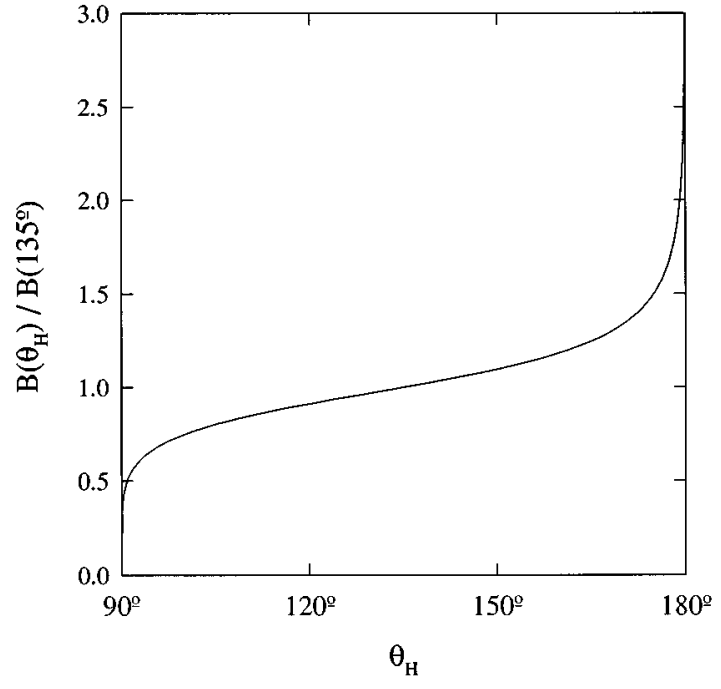

FIG. 4. Angular dependence of the tunneling exponent.

Equation (19) should be compared with Eq. (8) of Zaslavskii. ${ }^{21}$ Working out the correspondence of his parameters $B, C, B_{0}, \delta$ to our variables $S, \epsilon$, and $\theta_{H}$ we find that the two expressions coincide ${ }^{24}$ if one replaces $S+1 / 2$ in Zaslavskii's formula by $S$, which is justified in the limit of $S \gg 1$.

The angular dependence of $B$ is plotted in Fig. 4. We see that it has a rather unexpected distinct dependence on the orientation of the field. It is almost flat for $\theta_{H}$ not close to $90^{\circ}$ or $180^{\circ}$, rises sharply as $\theta_{H}$ approaches $180^{\circ}$ in accordance with the fact that at $\theta_{H}=180^{\circ} M_{z}$ commutes with the Hamiltonian, and rapidly drops at $\theta_{H} \rightarrow 90^{\circ}$. The latter result must be taken with caution because at $\theta_{H}=90^{\circ}$ the problem possesses a symmetry that has not been explicitly taken into account. It becomes the problem of the tunneling between equivalent minima studied, e.g., in Refs. 1,3. In this case the potential is of the form $\delta^{2}-\delta^{4}$ and the answer for $B$ reads $^{3}$ $B=4 S \epsilon^{3 / 2}$. Consequently, the result (19) holds for $\theta_{H}=90^{\circ}+\beta$, where $\beta$ is large compared to $\epsilon^{3 / 2}$. This means that for, e.g., $\epsilon=10^{-2}$, Eq. (19) is certainly true outside the $1^{\circ}$ vicinity of $90^{\circ}$. Of course, one should remember that all our conclusions on the angular and field dependence of tunneling are only valid in the limit of low barrier. For a particle of a considerable size, this is the only limit accessible experimentally. The angular dependence in general case is unknown.

Finally one should analyze the validity of the semiclassical approximation. Obviously, for the method to be valid, the tunneling probability must be small; that is, $B \gg 1$ is required. This may not be enough, however. One should also worry that the energy $\hbar \omega$ of zero-point oscillations at the bottom of the metastable well is sufficiently small compared to the height of the barrier, $U$. As follows from Eq. (16), the zero-point energy is $\hbar \omega=2 \hbar \omega_{0}$. The barrier height can be computed from Eq. (9),

$$
U=\hbar \omega_{a} S\left(\frac{2 \epsilon}{3}\right)^{3 / 2} \sin 2 \theta_{c}
$$

Working out the ratio, we obtain 


$$
\frac{U}{\hbar \omega}=\frac{5}{36} B
$$

The optimal range of $B$ in experiments on single particles should be $B \sim 30-35$ (see Sec. V). In this case the semiclassical approximation should be already rather good. In the case of a large number of identical tunneling objects (e.g., $M n_{12}$ molecules), the long lifetime of a metastable state should be of lesser concern, so that greater values of $B$ could be allowed. The corresponding conditions on $S$ and $\epsilon$ to be used in experiment will be discussed in Sec. V.

\section{TUNNELING RATE AND THE CROSSOVER TO THERMAL REGIME}

We shall now discuss the value of the preexponential factor $A$ in $\Gamma=A e^{-B}$. Contrary to $B$, it is sensitive to the structure of quantum levels in the potential shown in Fig. 2. If the potential to the right from the escape point $\theta_{2}$ was unbounded from below, tunneling would occur into the continuum of states. In this case the prefactor for the potential $\delta^{2}-\delta^{3}$ (our low barrier limit) is known (see, e.g., Ref. 4):

$$
A=\left(\frac{15}{2 \pi}\right)^{1 / 2} B^{1 / 2} \omega_{0}
$$

However, the potential given by Eq. (3), as well as the full potential (2), is certainly bounded. Correspondingly, the tunneling occurs onto the quantized quasilevels in the right well. Physically this means that the magnetic moment, after tunneling through the barrier, precesses about the direction of the effective field, $\vec{H}-H_{a} \hat{e}_{z}$, with the frequency of the precession being quantized. Of course, the same problem exists for a particle in an asymmetric double well. However, for a spin, unlike a particle, the two wells are intrinsically bounded. The only attempt to approach this problem quantitatively, known to the authors, has been made by Garg. ${ }^{9} \mathrm{He}$ argued that the answer depends crucially on the width of the excited levels in the right well. If the width is sufficiently large, so that the levels overlap, the problem is more or less equivalent to the tunneling into the structureless continuum. In this case, of course, Eqs. (19) and (22) must be altered for dissipation in the spirit of Caldeira and Leggett. ${ }^{4}$ Strong dissipation is hardly the case for magnetic systems, ${ }^{5-9}$ so that Eq. (19) is expected to hold and Eq. (22) is expected to give the correct order of magnitude for the prefactor. In the case of narrow levels, the tunneling rate at zero temperature becomes $^{9}$

$$
\Gamma=\sum_{n} \Delta_{0 n}^{2} \frac{\gamma_{n}}{\left(E_{0}-E_{n}\right)^{2}+\gamma_{n}^{2} / 4},
$$

where $\Delta_{0 n}$ is the WKB amplitude of the transition from the left-well level $E_{0}$ onto the right-well level $E_{n}$, and $\gamma_{n}$ is the width of the $E_{n}$ level. In this case Eq. (19) must give the correct answer for the exponent but the prefactor is totally different. It has been argued ${ }^{25,26,9}$ that it should oscillate on the applied field. It is not clear, however, what should be the effect of finite temperature on these findings. The full analysis of spin tunneling onto the precession levels remains an open problem.

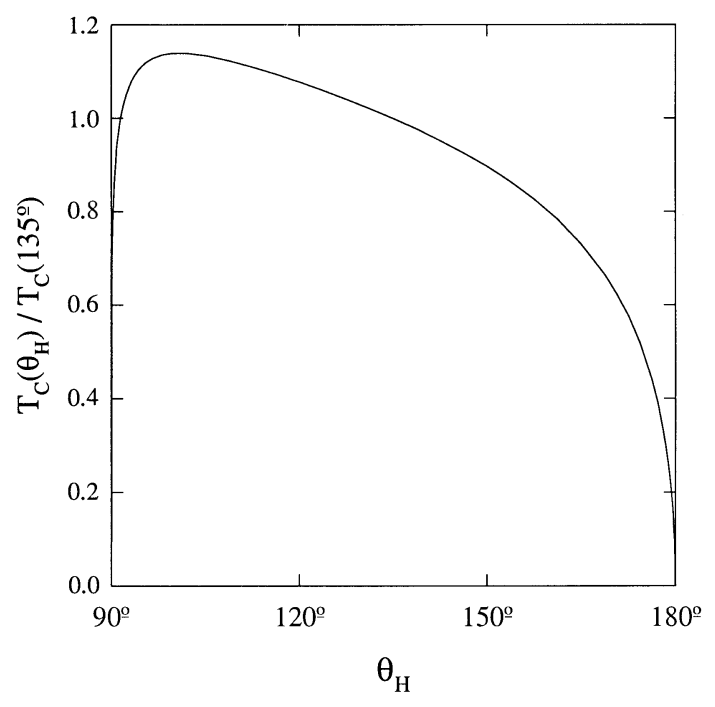

FIG. 5. The dependence of the crossover temperature on the orientation of the magnetic field.

Let us now concentrate on the transition exponent which is usually addressed by experiments. At a sufficiently large temperature it must cross to the Boltzmann exponent $U / T$. Then, equating $B$ of Eq. (19) to $U / T$, where $U$ is given by Eq. (20), we obtain that the crossover from the quantum to the thermal regime occurs at

$$
T_{c}=\frac{5}{18} \hbar \omega_{0},
$$

where we have used Eqs. (6) and (7). Another method ${ }^{27}$ to obtain the crossover temperature is based upon the analysis of the $\phi(\tau), \theta(\tau)$ solutions of Eq. (12) which are periodic in $\tau$ with the period $\hbar / T$. At finite temperature only such trajectories contribute to the path integral. Equations (12) on a class of periodic trajectories have a bifurcation at $T_{c}^{\prime}=\hbar \omega / 2 \pi$ where $\omega$ is the frequency of small oscillations near the bottom of the inversed potential in Fig. 2. Above this temperature the only trajectory of interest which is formally periodic with the period $\hbar / T$ is a static solution of Eq. (12): $\theta=\theta_{1}, \phi=0$. It corresponds to $\vec{M}$ at the top of the potential barrier. Substitution of this solution into Eq. (11) gives $B=U / T$. A simple analysis yields $\omega=2 \omega_{0}$, that is,

$$
T_{c}^{\prime}=\frac{1}{\pi} \hbar \omega_{0} .
$$

The comparison of Eq. (24) and Eq. (25) shows that they differ by a factor $\sim 1.15$, and, therefore, can both be used as the definition of the crossover temperature.

Because of the exponential dependence of the thermal rate on temperature the transition from thermal to quantum regime, as the temperature is lowered, must be rather sharp, with a well-defined crossover. The dependence of $T_{c}$ on $\theta_{H}$ is plotted in Fig. 5. The plot suggests that the observation of the quantum decay of a metastable state at $\theta_{H} \rightarrow 90^{\circ}$ and $\theta_{H} \rightarrow 180^{\circ}$ requires much lower temperatures than for intermediate orientations of the field. Again, the vicinity of $90^{\circ}$ must be treated with caution. In that region $T_{c}$ does not, in fact, go to zero but is proportional to the higher power of a 
small parameter $\epsilon,{ }^{3} \epsilon^{1 / 2}$ instead of $\epsilon^{1 / 4}$ for intermediate angles. Writing $\theta_{H}$ as $90^{\circ}+\beta$ we again find that our result for $T_{c}$ is correct for $\beta>\epsilon^{3 / 2}$, that is, quite close to $90^{\circ}$ for $\epsilon \leqslant 10^{-2}$.

\section{CONCLUSIONS AND SUGGESTIONS FOR EXPERIMENT}

We have studied quantum decay of a metastable magnetic state of a uniaxial single-domain particle placed at a some angle with the magnetic field. Our interest in this problem has been stimulated by the fact that the corresponding experiment would be most easy to perform and to interpret. It gives the experimentalist three control parameters for comparison with theory: the orientation of the field $\theta_{H}$, the field strength $\epsilon=1-H / H_{c}$, and the temperature $T$. According to Eqs. (18), (23), and (24), the crossover temperature is proportional to the anisotropy field. Thus, selecting a particle with $H_{a} \geqslant 1 T$ would ensure that a reasonably low temperature is required. A weak dependence of $T_{c}$ on $\epsilon, T_{c} \propto \epsilon^{1 / 4}$, works to the advantage of an experimentalist. For, e.g., $H_{a}=1 T$ and $\epsilon=10^{-3}$ we obtain $T_{c}\left(135^{\circ}\right) \sim 30 \mathrm{mK}$. Note that $\epsilon \sim 10^{-3}$ requires that the field be controlled with an accuracy higher than $H_{a} \epsilon$, that is, better than 10 Oe for $H_{a} \sim 1 \mathrm{~T}$. This poses no problem.

If the experiment is to be performed with a large number of identical magnetic entities, the long lifetime of a metastable state will not necessarily interfere with the possibility to detect the effect (compare with radioactive decay). In the case of a single particle, however, a very long, as well as very short, lifetime can be a serious obstacle, so that special consideration should be given to the selection of the appropriate size of the particle. For $H_{a} \sim 1 \mathrm{~T}$ the prefactor of Eq. (22) is of the order of $10^{11} \mathrm{~s}^{-1}$. Consequently, the tunneling exponent which is significantly less than 30 will make tunneling very fast and, therefore, difficult to observe. It will also invalidate the semiclassical approximation employed in this paper, as has been discussed at the end of Sec. III. On the other hand, for $B$ greater than 30-35 the lifetime of the metastable state may significantly exceed the time of the experiment. Thus, $B \sim 30-35$ seems to be optimal for the observation of tunneling and, at the same time, for remaining in the domain of MQT . According to (19), this means that at $\epsilon \sim 10^{-3}$ the total spin of the particle $S$ should be no more than $3 \times 10^{4}$. This certainly falls in the domain of MQT. Note that even for $\epsilon$ as small as $10^{-3}$ the underbarrier rota- tion of $\vec{M}$ corresponds to an appreciable change of the orientation, $\delta_{2}=\sqrt{6 \epsilon} \mathrm{rad}>4^{\circ}$. Greater $\epsilon$ will give a larger angle and a higher $T_{c}$ but will move the maximal allowed $S$ to lower values. Smaller $\epsilon$ are dangerous because of the interaction of $\vec{M}$ with nuclear spins. ${ }^{7}$

We suggest the following experimental procedure. First, the anisotropy axis of the particle must be precisely determined by allowing it to orient freely in the magnetic field, or by other methods. The position of the particle must then be fixed at a certain angle $\theta_{H}$ with the field; see Fig. 1 . The magnitude of $H$ should then be slowly increased to obtain the critical field $H_{c}\left(\theta_{H}\right)$ at which the barrier dissapears. The metastable state should then be created again and the field should be tuned to the value $H_{c}(1-\epsilon)$ just below $H_{c}$. By repeating this procedure many times the transition rate must be obtained. The field orientation then should be changed and the whole procedure repeated for different values of $\theta_{H}$ and $H_{c}\left(\theta_{H}\right)$ but with the same value of $\epsilon$. This would give the transition rate as a function of $\theta_{H}$, which can be compared with the theoretical prediction given by Eq. (19) and illustrated in Fig. 4. To ensure that quantum, not ther$\mathrm{mal}$, transitions are measured, the temperature independence of the rate should be checked at any $\theta_{H}$.

The above experiment requires very high sensitivity if it has to be performed with a single particle, or it requires identical particles if it has to be performed with an ensemble of particles. The latter is difficult to achieve for singledomain particles but comes naturally if one takes big magnetic molecules instead. In the last years evidence of spin tunneling has been reported in $M n_{12}$ molecular complexes having very weakly interacting magnetic entities of spin $S=10 .{ }^{14-17}$ The system is uniaxial with high accuracy. The reported anisotropy field is very high, $H_{a} \sim 10 \mathrm{~T}$. This makes it a good candidate for the experimental study suggested above, though we cannot guarantee our theory to work well for the spin as low as 10 . Some correlations between the theory and experiment should exist, however. One of them may be the weak dependence of the tunneling rate on the orientation of the field observed in $M n_{12} \cdot{ }^{28}$

\section{ACKNOWLEDGMENTS}

M.C.M. acknowledges support from the Generalitat de Catalunya. The work of E.M.C. has been supported by the U.S. National Science Foundation through Grant No. DMR9024250 .
*On sabbatical leave from Physics Department, CUNY Lehman College, Bronx, NY 10468-1589.

${ }^{1}$ J.L. van Hemmen and A. Sütö, Physica B 141, 37 (1986); Europhys. Lett. 1, 481 (1986); J.L. van Hemmen and W.F. Wreszinski, Commun. Math. Phys. 119, 213 (1988).

${ }^{2}$ M. Enz and R. Schilling, J. Phys. C 19, 1765 (1986); 19, L711 (1986)

${ }^{3}$ E.M. Chudnovsky and L. Gunther, Phys. Rev. Lett. 60, 661 (1988); Phys. Rev. B 37, 9455 (1988).

${ }^{4}$ A.O. Caldeira and A.J. Leggett, Phys. Rev. Lett. 46, 211 (1981); Ann. Phys. (N.Y.) 149, 374 (1983).

${ }^{5}$ Anupam Garg and Gwang-Hee Kim, Phys. Rev. Lett. 63, 2512 (1989); Phys. Rev. B 43, 712 (1991).
${ }^{6}$ P.C.E. Stamp, E.M. Chudnovsky, and B. Barbara, Int. J. Mod. Phys. 6, 1355 (1992).

${ }^{7}$ Anupam Garg, Phys. Rev. Lett. 70, 1541 (1993).

${ }^{8}$ E.M. Chudnovsky, Phys. Rev. Lett. 72, 3433 (1994).

${ }^{9}$ Anupam Garg, Phys. Rev. B 51, 15161 (1995).

${ }^{10}$ B. Barbara et al., J. Appl. Phys. 73, 6703 (1993) and references therein; J. Magn. Magn. Mater. 140-144, 1825 (1995) and references therein.

${ }^{11}$ J. Tejada, X.X. Zhang, and Ll. Balcells, J. Appl. Phys. 73, 6709 (1993) and references therein; J. Tejada and X.X. Zhang, J. Magn. Magn. Mater. 140-144, 1815 (1995) and references therein.

${ }^{12}$ D.D. Awschalom et al., Phys. Rev. Lett. 68, 3092 (1992). 
${ }^{13}$ W. Wernsdorfer et al., J. Magn. Magn. Mater. 140-144, 385 (1995).

${ }^{14}$ C. Paulsen et al., J. Magn. Magn. Mater. 140-144, 379 (1995).

${ }^{15}$ P. Politi et al., Phys. Rev. Lett. 75, 537 (1995).

${ }^{16}$ M.A. Novak and R. Sessoli, in Quantum Tunneling of Magnetization - QTM '94, edited by L. Gunther and B. Barbara (Kluwer Academic, Dordrecht/Boston/London, 1995).

${ }^{17}$ J.R. Friedman, M. P. Sazachik, J. Tejada, and R. Ziolo, Phys. Rev. Lett. 76, 3830 (1996).

${ }^{18}$ D.P. DiVincenzo, in Quantum Tunneling of Magnetization QTM '94, edited by L. Gunther and B. Barbara (Kluwer Academic, Dordrecht/Boston/London, 1995).

${ }^{19}$ D. Loss, D.P. DiVincenzo, and G. Grinstein, Phys. Rev. Lett. 69, 3232 (1992).

${ }^{20}$ J. von Delft and C.L. Henley, Phys. Rev. Lett. 69, 3236 (1992).

${ }^{21}$ O.B. Zaslavskii, Phys. Rev. B 42, 992 (1990).
${ }^{22}$ G. Sharf, Ann. Phys. (N.Y.) 83, 71 (1974); Helv. Phys. Acta 48, 329 (1975); O.B. Zaslavskii and V.V. Ulyanov, Zh. Éksp. Teor. Fiz. 87, 1724 (1984) [Sov. Phys. JETP 60, 991 (1984)].

${ }^{23}$ A.H. Morrish, The Physical Principles of Magnetism (Krieger, Malabar, FL, 1983).

${ }^{24}$ Actually, there is a difference by a factor of 9 between Zaslavskii's and our formulas. We carefully checked our calculation and found no mistake. Since all other factors coincide precisely, we attribute the difference to a lost factor in Ref. 21, which is difficult to trace because of the chain of redefinitions going back to Ref. 22.

${ }^{25}$ Anupam Garg, Europhys. Lett. 22, 205 (1993).

${ }^{26}$ E.M. Chudnovsky and D.P. DiVincenzo, Phys. Rev. B 48, 10548 (1993).

${ }^{27}$ I. Affleck, Phys. Rev. Lett. 46, 388 (1981).

${ }^{28} \mathrm{C}$. Paulsen (private communication). 